PROCEEDINGS OF THE

AMERICAN MATHEMATICAL SOCIETY

Volume 135, Number 4, April 2007, Pages 1163-1168

S 0002-9939(06)08569-8

Article electronically published on October 13, 2006

\title{
SHARP BEREZIN LIPSCHITZ ESTIMATES
}

\author{
L. A. COBURN
}

(Communicated by Joseph A. Ball)

\begin{abstract}
F.A. Berezin introduced a general "symbol calculus" for linear operators on reproducing kernel Hilbert spaces. For the Segal-Bargmann space $H^{2}\left(\mathbf{C}^{n}, d \mu\right)$ of Gaussian square-integrable entire functions on complex $n$-space, $\mathbf{C}^{n}$, or for the Bergman spaces $A^{2}(\Omega)$ of Euclidean volume square-integrable holomorphic functions on bounded domains $\Omega$ in $\mathbf{C}^{n}$, we show here that earlier Lipschitz estimates for Berezin symbols of arbitrary bounded operators are sharp.
\end{abstract}

\section{INTRODUCTION}

The Segal-Bargmann Hilbert space $H^{2}\left(\mathbf{C}^{n}, d \mu\right)$ of Gaussian square-integrable entire functions on complex $n$-space [2] has the Bergman "reproducing kernel" property that

$$
f(a)=\int_{\mathbf{C}^{n}} K(a, z) f(z) d \mu(z)=\langle f(\cdot), K(\cdot, a)\rangle
$$

for all $f$ in $H^{2}\left(\mathbf{C}^{n}, d \mu\right)$ and $a$ in $\mathbf{C}^{n}$ where $a \cdot z=a_{1} \bar{z}_{1}+\cdots+a_{n} \bar{z}_{n},|a|^{2}=a \cdot a$, $K(a, z)=e^{a \cdot z / 2}$ is the Bergman kernel function and

$$
d \mu(z)=(2 \pi)^{-n} \exp \left\{-|z|^{2} / 2\right\} d v(z)
$$

( $d v$ is Lebesgue volume measure). It is easy to check that

$$
k_{a}(z)=K(z, a)\{K(a, a)\}^{-\frac{1}{2}}
$$

is a unit vector in the Hilbert space structure that $H^{2}\left(\mathbf{C}^{n}, d \mu\right)$ inherits as a subspace of $L^{2}\left(\mathbf{C}^{n}, d \mu\right)$.

Although we use the space $H^{2}\left(\mathbf{C}^{n}, d \mu\right)$ as a model, we will also consider the analogous Bergman spaces $A^{2}(\Omega)$ for $\Omega$ a bounded domain in $\mathbf{C}^{n}$ and the Gaussian $d \mu$ replaced by $d v$. Their kernel functions will also be denoted by $K(a, z)$ as in [15, pp. 39-54]. Note that $K(a, z)$ is always analytic in $a$ and conjugate-analytic in $z$ with

$$
\overline{K(a, z)}=K(z, a)
$$

and that $K(a, a)$ is positive. The "bounded symmetric domains" $\Omega$ give interesting special cases which are "closest" to the model $H^{2}\left(\mathbf{C}^{n}, d \mu\right)$.

For bounded linear operators $X$ on $H^{2}\left(\mathbf{C}^{n}, d \mu\right)$ or $A^{2}(\Omega)$, Berezin $[3$, 4 considered the mapping Ber $: X \rightarrow \tilde{X}$, where $\tilde{X}(a)=\left\langle X k_{a}, k_{a}\right\rangle$. The function $\tilde{X}(\cdot)$ is real-analytic on $\mathbf{C}^{n}$ or $\Omega$ and is uniquely determined by $X$ [3; [11, pp. 43, 139].

Received by the editors October 18, 2005 and, in revised form, November 15, 2005.

2000 Mathematics Subject Classification. Primary 47B32; Secondary 32A36.

(C)2006 American Mathematical Society 
It is clear that $|\tilde{X}(\cdot)| \leq\|X\|$, where $\|\cdot\|$ is the usual operator norm. Because Ber is linear and one-to-one, it "encodes" operator-theoretic information into function theory in a striking but somewhat impenetrable way.

In fact, since $k_{a} \rightarrow 0$ weakly as $|a| \rightarrow \infty$ for $H^{2}\left(\mathbf{C}^{n}, d \mu\right)$, it is also clear that Ber maps compact operators on these Hilbert spaces into functions which vanish at infinity (there is an analogous result for $A^{2}(\Omega)$ with "nice" $\Omega$ ). Because of these properties, the mapping Ber has found useful applications in dealing with operators "of function-theoretic significance" such as Toeplitz and Hankel operators [1, [5, [6], 7], [12, 9]. The functions $\tilde{X}$ also arise in Berezin's well-known quantization program [4]. It is, therefore, of interest to determine the range of Ber.

In this note, we consider the infinitesimal Bergman metric on $\Omega$ ( or on $\mathbf{C}^{n}$ ) defined by writing

$$
g_{j, k}(z)=2 \frac{\partial}{\partial \bar{z}_{j}} \frac{\partial}{\partial z_{k}} \ln K(z, z)
$$

(the constant "2" conforms with [16]). It is well known [14 that (*) gives a Riemannian metric on $\Omega$ (it is just the usual Euclidean metric on $\mathbf{C}^{n}$ ). Following [16], we define the Bergman length of a $C^{1}$-curve $\gamma:[0,1] \rightarrow \Omega$ by

$$
l(\gamma)=\int_{0}^{1}\{G(s)\}^{\frac{1}{2}} d s
$$

where

$$
G(s)=\sum_{j, k=1}^{n} g_{j k}(\gamma(s)) \overline{\gamma_{j}^{\prime}(s)} \gamma_{k}^{\prime}(s) .
$$

The Bergman metric distance function $\beta(a, b)$ is defined by

$$
\beta(a, b)=\inf \{l(\gamma): \gamma(0)=b, \gamma(1)=a\} .
$$

In $\left[8\right.$, the following estimates for all bounded domains $\Omega$ and for $\mathbf{C}^{n}$ were obtained:

$$
\begin{aligned}
|\tilde{X}(a)-\tilde{X}(b)| & \leq 2\|X\|\left\{1-\left|\left\langle k_{a}, k_{b}\right\rangle\right|^{2}\right\}^{\frac{1}{2}} \\
& \leq \sqrt{2}\|X\| \beta(a, b) .
\end{aligned}
$$

In the next two sections, we show, by varying $X, a, b$, that these estimates are sharp.

\section{Preliminary RESUlts}

For the remainder of this note, we pick $\gamma$ to be a geodesic curve for the Bergman metric, emanating from $b$ in $\Omega$ with $\gamma(0)=b$ and with $\gamma^{\prime}(0)=v$, a fixed unit vector in $\mathbf{C}^{n}$. It is then clear from Theorem 9.9 of [14, p. 53] that, for some $t_{0}>0$ and $0<t<t_{0}$,

$$
\beta(\gamma(t), b)=\int_{0}^{t}\{G(s)\}^{\frac{1}{2}} d s
$$

and, by the fundamental theorem of the integral calculus,

$$
\lim _{t \rightarrow 0} \frac{\beta(\gamma(t), b)}{t}=\{G(0)\}^{\frac{1}{2}}>0 .
$$


As in [8, consider the rank-one projection $P_{a}(f)=\left\langle f, k_{a}\right\rangle k_{a}$ onto the span of $k_{a}$. We will use some standard results from [13] about trace-class operators. Recall that

$$
\tilde{X}(a)=\operatorname{trace}\left(X P_{a}\right) .
$$

A slight elaboration of Theorem 1 of 8 yields

Proposition 1. The rank-two self-adjoint operator $P_{a}-P_{b}$ has real eigenvalues $\{\lambda,-\lambda\}$ where $\lambda \geq 0$ with $\lambda=\left\{1-\left|\left\langle k_{a}, k_{b}\right\rangle\right|^{2}\right\}^{\frac{1}{2}}$. It follows that the operator norm of $P_{a}-P_{b}$ is given by

$$
\left\|P_{a}-P_{b}\right\|=\left\{1-\left|\left\langle k_{a}, k_{b}\right\rangle\right|^{2}\right\}^{\frac{1}{2}},
$$

while the trace norm of $P_{a}-P_{b}$ is given by

$$
\left\|P_{a}-P_{b}\right\|_{\text {trace }}=2\left\{1-\left|\left\langle k_{a}, k_{b}\right\rangle\right|^{2}\right\}^{\frac{1}{2}} .
$$

Proof. Since $P_{a}, P_{b}$ are both rank-one self-adjoint operators with

$$
\operatorname{trace}\left(P_{a}\right)=\operatorname{trace}\left(P_{b}\right)=1,
$$

it follows that there is an orthonormal basis in which $P_{a}-P_{b}$ is diagonal with two (possibly) nonzero real eigenvalues $\{\lambda,-\lambda\}$ and $\lambda \geq 0$. It follows that $\left(P_{a}-P_{b}\right)^{2}$ has nonzero eigenvalues $\left\{\lambda^{2}, \lambda^{2}\right\}$. On the other hand, it is easy to calculate

$$
\begin{aligned}
\operatorname{trace}\left(P_{a}-P_{b}\right)^{2} & =\operatorname{trace}\left(P_{a}-P_{a} P_{b}-P_{b} P_{a}+P_{b}\right) \\
& =2-2 \operatorname{trace}\left(P_{a} P_{b}\right) \\
& =2-2\left\langle P_{a} P_{b} k_{b}, k_{b}\right\rangle \\
& =2-2\left\langle P_{a} k_{b}, k_{b}\right\rangle \\
& =2-2\left\langle k_{b}, k_{a}\right\rangle\left\langle k_{a}, k_{b}\right\rangle \\
& =2\left\{1-\left|\left\langle k_{a}, k_{b}\right\rangle\right|^{2}\right\} .
\end{aligned}
$$

It follows that $\lambda^{2}=\left\{1-\left|\left\langle k_{a}, k_{b}\right\rangle\right|^{2}\right\}$ and the proposition follows easily.

For the Skwarczynski distance function

$$
\rho(a, b)=\left\{1-\left|\left\langle k_{a}, k_{b}\right\rangle\right|\right\}^{\frac{1}{2}},
$$

[16] shows that

$$
\lim _{t \rightarrow 0} \frac{\rho(\gamma(t), b)}{t}=\frac{1}{2}\{G(0)\}^{\frac{1}{2}} .
$$

We now have the key technical result:

Proposition 2. For $\gamma$ a Bergman metric geodesic, as above, we have

$$
\begin{aligned}
\lim _{t \rightarrow 0} \frac{\left\|P_{\gamma(t)}-P_{b}\right\|_{\text {trace }}}{t} & =\sqrt{2}\{G(0)\}^{\frac{1}{2}} \\
& =\sqrt{2} \lim _{t \rightarrow 0} \frac{\beta(\gamma(t), b)}{t} .
\end{aligned}
$$

Proof. From Proposition 1, we have

$$
\left\|P_{a}-P_{b}\right\|_{\text {trace }}=2\left\{1+\left|\left\langle k_{a}, k_{b}\right\rangle\right|\right\}^{\frac{1}{2}} \rho(a, b) .
$$

It follows that

$$
\lim _{t \rightarrow 0} \frac{\left\|P_{\gamma(t)}-P_{b}\right\|_{\text {trace }}}{t}=2 \lim _{t \rightarrow 0}\left\{1+\left|\left\langle k_{\gamma(t)}, k_{b}\right\rangle\right|\right\}^{\frac{1}{2}} \lim _{t \rightarrow 0} \frac{\rho(\gamma(t), b)}{t} .
$$


It is easy to check that

$$
\lim _{t \rightarrow 0}\left\{1+\left|\left\langle k_{\gamma(t)}, k_{b}\right\rangle\right|\right\}^{\frac{1}{2}}=\sqrt{2},
$$

and the rest follows from $(\dagger),(\dagger \dagger)$.

\section{SHARPNESS OF THE ESTIMATES}

To obtain the promised sharpness results, we need to consider a family of "optimal" bounded operators $X_{t}$. This has been detected by a process of trial and error which is best left unexamined. For $\gamma(t)$ a Bergman metric geodesic as above, with $\gamma(0)=b$ and $\gamma^{\prime}(0)=v$, a unit vector, we consider the family of operators

$$
X_{t}=P_{\gamma(t)}-P_{b} .
$$

Note that, by Proposition 1 ,

$$
\left\|X_{t}\right\|=\left\{1-\left|\left\langle k_{\gamma(t)}, k_{b}\right\rangle\right|^{2}\right\}^{\frac{1}{2}} .
$$

We can also calculate easily that

$$
\tilde{X}_{t}(\gamma(t))-\tilde{X}_{t}(b)=2\left\{1-\left|\left\langle k_{\gamma(t)}, k_{b}\right\rangle\right|^{2}\right\} .
$$

Again using Proposition 1, we have

$$
\left\|P_{\gamma(t)}-P_{b}\right\|_{\text {trace }}=2\left\{1-\left|\left\langle k_{\gamma(t)}, k_{b}\right\rangle\right|^{2}\right\}^{\frac{1}{2}} .
$$

We now obtain

Sharpness Theorem 1. For $X_{t}$ as above,

$$
\frac{\left|\tilde{X}_{t}(\gamma(t))-\tilde{X}_{t}(b)\right|}{\left\|X_{t}\right\|\left\|P_{\gamma(t)}-P_{b}\right\|_{\text {trace }}} \equiv 1
$$

while

$$
\lim _{t \rightarrow 0} \frac{\left|\tilde{X}_{t}(\gamma(t))-\tilde{X}_{t}(b)\right|}{\left\|X_{t}\right\| \beta(\gamma(t), b)}=\sqrt{2} .
$$

Proof. (a) is a direct calculation. Proposition 2 yields

$$
\begin{aligned}
\lim _{t \rightarrow 0} \frac{\left\|P_{\gamma(t)}-P_{b}\right\|_{\text {trace }}}{\beta(\gamma(t), b)} & =\frac{\lim _{t \rightarrow 0} \frac{\left\|P_{\gamma(t)}-P_{b}\right\|_{\text {trace }}}{t}}{\lim _{t \rightarrow 0} \frac{\beta(\gamma(t), b)}{t}} \\
& =\sqrt{2} .
\end{aligned}
$$

This, with (a), yields (b).

Remark. The sharpness of $(* *)$ provides striking evidence of the efficiency of the trace estimates of [13, pp. 47, 48, 92].

\section{OPEN PROBLEMS AND REMARKS}

For bounded domains $\Omega$ in $\mathbf{C}^{n}$, and the special case of operators $X$ of "multiplication by a bounded analytic function $\varphi$ ", 8 , provided a Bloch-type estimate for all $a, b$ in $\Omega$ :

$$
|\varphi(a)-\varphi(b)| \leq \sqrt{2}\|\varphi\|_{\infty} \beta(a, b) .
$$


Problem 1. Determine the sharp constants $B_{\Omega}$ for which

$$
|\varphi(a)-\varphi(b)| \leq B_{\Omega}\|\varphi\|_{\infty} \beta(a, b)
$$

for all bounded analytic $\varphi$ on $\Omega$ and all $a, b$ in $\Omega$.

From (†††), by choosing $\varphi$ in the set of coordinate functions $\left\{z_{j}: j=1,2,3, \ldots, n\right\}$, we see that for each bounded $\Omega$ there is a constant $C_{\Omega}$ with

$$
|a-b| \leq C_{\Omega} \beta(a, b)
$$

for all $a, b$ in $\Omega$.

Problem 2. Determine sharp values of $C_{\Omega}$.

Remark. Jingbo Xia has pointed out that the proof of Theorem 4 of $[8$ can be used, along with a direct calculation, to provide a strengthened version of Theorem 2 of [8]: for any $X$ in the full algebra of bounded operators $O p\left\{H^{2}\left(\mathbf{C}^{n}, d \mu\right)\right\}, \tilde{X}$ and its partial derivatives of all orders are bounded. Subsequently, Miroslav Engliš and Genkai Zhang [10 have provided the appropriate generalization of this result for invariant differential operators applied to $\tilde{X}$ on bounded symmetric $\Omega$.

\section{Dedication}

I dedicate this note to the memory of my late wife Charlaine Ackerman Coburn (1939-2005). She was sharp and constant.

\section{ACKNOWLEDGMENTS}

I thank Bo Li, Mohan Ramachandran and Jingbo Xia for useful discussions.

\section{REFERENCES}

[1] S. Axler and D. Zheng, Compact operators via the Berezin transform, Indiana Univ. Math. J., 47 (1998), 387-400. MR1647896 (99i:47045)

[2] V. Bargmann, On a Hilbert space of analytic functions and an associated integral transform, Communications on Pure and Applied Mathematics, 14 (1961), 187-214. MR0157250 (28:486)

[3] F. A. Berezin, Covariant and contravariant symbols of operators, Math. USSR Izv., 6 (1972), 1117-1151. MR0350504 (50:2996)

[4] F. A. Berezin, Quantization, Math. USSR Izv., 8 (1974), 1109-1163. MR0395610 $(52: 16404)$

[5] D. Bèkollè, C. A. Berger, L. A. Coburn, and K. H. Zhu, BMO in the Bergman metric on bounded symmetric domains, J. Funct. Anal., 93 (1990), 310-350. MR1073289 (91j:32034)

[6] C. A. Berger and L. A. Coburn, Toeplitz operators on the Segal-Bargmann space, Transactions AMS, 301 (1987), 813-829. MR0882716 (88c:47044)

[7] C. A. Berger and L. A. Coburn, Heat flow and Berezin-Toeplitz estimates, American Journal of Mathematics, 116 (1994), 563-590. MR1277446 (95g:47038)

[8] L. A. Coburn, A Lipschitz estimate for Berezin's operator calculus, Proceedings of the American Mathematical Society, 133 (2005), 127-131. MR2085161 (2005e:47060)

[9] M. Engliš, Compact Toeplitz operators via the Berezin transform on bounded symmetric domains, Integral Equations Operator Theory, 33 (1999), 426-455. MR1682815 (2000h:47050a)

[10] M. Engliš and G. Zhang, On the derivatives of the Berezin transform, Proceedings of the American Mathematical Society, 134 (2006), 2285-2294.

[11] G. B. Folland, Harmonic analysis in phase space, Annals of Mathematics Studies, Princeton Univ. Press, Princeton, 1989. MR0983366 (92k:22017)

[12] V. Guillemin, Toeplitz operators in $n$ dimensions, Integral Equations and Operator Theory, 7 (1984), 145-205. MR0750217 (86i:58130) 
[13] I. C. Gohberg and M. G. Krein, Introduction to the theory of linear nonselfadjoint operators, Translations of Mathematical Monographs 18, American Mathematical Society, Providence, 1969. MR0246142 (39:7447)

[14] S. Helgason, Differential geometry, Lie groups, and symmetric spaces, Academic Press, New York, 1978. MR0514561 (80k:53081)

[15] S. Krantz, Function theory of several complex variables, Wiley-Interscience, New York, 1982. MR0635928 (84c:32001)

[16] T. Mazur, P. Pflug and M. Skwarczynski, Invariant distances related to the Bergman function, Proceedings of the American Mathematical Society, 94 (1985), 72-76. MR0781059 (86i:32047)

Department of Mathematics, SUny at Buffalo, Buffalo, New York 14260

E-mail address: lcoburn@buffalo.edu 